\title{
CONCEPTUALIZACIÓN DEL APOYO SOCIAL Y LAS REDES DE APOYO SOCIAL
}

\author{
CONCEPTUALIZATION OF THE SOCIAL SUPPORT AND THE SOCIAL SUPPORT \\ NETWORK \\ Carolina Aranda B. ${ }^{1}$ y Manuel Pando M. \\ Instituto de Investigación en Salud Ocupacional, Departamento de Salud Pública, \\ Universidad de Guadalajara, MèXICO \\ (RECIBIDO 15/03/2013 - ACEPTADO 20/06/2013)
}

\section{RESUMEN}

Numerosos son los conceptos y abordajes que se han propuesto en el estudio del apoyo social como de las redes de apoyo social, así como del empleo de algunos de estos conceptos para relacionar bien sea el apoyo social o las redes de apoyo social a las condiciones de salud y su importancia en el papel protector de la misma. El recorrido sobre la conceptualización de ambas variables parte de los años cincuenta hasta lo más actual, cerrando con unas breves conclusiones

Palabras clave: apoyo social, red social de apoyo, salud, enfermedad

\begin{abstract}
Many are the concepts and approaches whose have been proposed in the study of the social support as social support networks, as well as the use of some of these concepts to relate either social support or social support networks to the conditions and its importance in the protective role of it. The route on the conceptualization of both variables occurs since the fifties to the most current, closing with some brief conclusions.
\end{abstract}

Keywords: social support, social support network, health, disease 


\section{INTRODUCCIÓN}

Muchos años han pasado desde que los conceptos sobre "apoyo social" y "redes de apoyo social" surgieron en la literatura con John Bowly en la década de los sesenta así como con John Barnes en los cincuenta. En realidad contar con redes de apoyo social tiene, además de los beneficios que se mencionarán en el resto del documento, un impacto significativo en la calidad de vida de las personas, y como refiere uno de ellos, para toda la vida.

\section{Década de los cincuenta}

El surgimiento del concepto de red de apoyo social nace con John Barnes en el año de 1954 tras la necesidad de explicar aspectos como "el acceso a puestos de trabajo, la actividad política y los roles maritales", para el año de 1955, Bott Elizabeth, describía a la red social como "una configuración social en la que algunas unidades externas que la componen mantienen relaciones entre sí", mientras que Caplow (1955) citado por González en el 2001, abarcaba también en sus estudios la variable de redes de apoyo social para su análisis.

\section{Década de los sesenta}

Los primeros conceptos sobre el apoyo social se originaron en los años sesenta por el psiquiatra británico John Bowly (1969) a través de su teoría del desapego, cuando surgió la necesidad de explicar el cariño de una madre hacia los hijos, en específico el del efecto protector, desde la infancia y durante la vida.

\section{Década de los setenta}

Tanto en la Gran Bretaña como en los Estados Unidos se daban a conocer la realización de diversos estudios en el que se incluía este concepto. En esta década se publican tres artículos pioneros en el tema con: Cassel J. $(1974,1976)$ sobre procesos psicosociales y estrés y, el trabajo de Cobb S. (1976) sobre el apoyo social como amortiguador de los procesos estresantes vitales. A partir de estos estudios es que el apoyo social se definiría como el proceso en que se dan las transacciones entre las personas (Caplan G., 1974; Cassel, Caplan y Cobb, 1976), o como "los vínculos entre individuos o entre individuos y grupos que sirven para mejorar la adaptación cuando uno se enfrenta a situaciones de estrés, reto o privación" (Caplan y Killilea, 1976) y, como "cualquier impulso provisto por otra persona (o grupo) que mueve al receptor hacia las metas que desea" (Caplan, Robinson, French, Caldwell y Shinn, 1976). Por su parte Cobb S. (1976) conceptúa por red social a la instancia mediadora en la que se brinda apoyo social de tipo emocional e informacional. Para el año de 1977 Kaplan, Cassel y Gores definían al apoyo social como "el grado de satisfacción de las necesidades sociales básicas (afecto, estima, pertenencia, etc.) a través de su interacción con otros individuos. Johnson y Sarason $(1979 a, b)$ definían al apoyo social como "el grado en que los individuos tienen acceso a recursos sociales, a partir de relaciones de confianza con otros individuos" y Lin, Simeone, Ensel y Kuo (1979) como el "apoyo accesible 
a un individuo a través de sus vínculos sociales con otros individuos, grupos y comunidad".

\section{Década de los ochenta}

En esta década, al menos encontramos veintidós estudios que hablan de ello, sin querer decir que sean los únicos. Iniciando con Maguire L. en 1980 en el que se entendería por red social a las "fuerzas preventivas que se brindan entre los individuos ante una situación crítica” y, con Kahn y Antonucci (1980-1981) a las transacciones que se dan entre las personas, las cuales implican ayuda, afecto o afirmación. Pearlin, Lieberman, Menaghan y Hullan (1981) lo definen como "el acceso y uso de individuos, grupos u organizaciones en relación con las vicisitudes de la vida". En ese mismo año Wellman B (1981) definía a la red social como un conjunto de lazos apoyadores, en donde se ofrecen transacciones entre ellas, tomando en cuenta la calidad, cantidad y distribución de recursos.

Un año más tarde Bell, LeRoy y Vitalo (1982) definían al apoyo social como "la actividad social y disponibilidad de amigos y familiares" y, Thoits P. (1982) como "el grado en que las necesidades sociales básicas (afiliación, afecto, pertenencia, identidad, seguridad y aprobación) son satisfechas a través de la interacción con otros". Israel BA (1982) considera que el apoyo social es aquel que se recibe de la interacción entre las personas para recibir y dar ayuda (espiritual, emocional, instrumental e informativo). Para Gottlieb B. (1983) el apoyo social es "la información verbal y no verbal, ayuda tangible o accesible dada por los otros o inferida por su presencia y que tiene efectos conductuales y emocionales beneficiosos en el receptor y, en el año de 1984 Berkman L. delimitaba a la red social "como un tipo específico de relaciones que vincula a un grupo determinado de personas, objetos y acontecimientos". Para Shumaker y Brownell (1984) el apoyo social es el "intercambio entre al menos dos individuos, con el objetivo percibido por el proveedor o el receptor, de incrementar el bienestar del receptor". Para el año de 1985, diversos autores describirían uno u otro concepto: 1. Shumaker y Brownell, 2. Cohen y Syme (a) y 3. Sarason, Sarason, Hacker y Basham. Los primeros autores refieren al apoyo social como el "contenido funcional de las interacciones sociales", los segundos como la "totalidad de recursos provistos por otras personas"; mientras que los últimos lo describían como aquel que "se relaciona con la calidad de vida e incluye el conjunto de provisiones expresivas o instrumentales (percibidas o recibidas) proporcionadas por la comunidad, las redes sociales y las personas de confianza, que se pueden producir tanto en situaciones cotidianas como de crisis".

Autores como Lin, Dean y Ensel (1986) definían por apoyo social al "conjunto de provisiones expresivas o instrumentales - percibidas o recibidas- proporcionadas por la comunidad, las redes sociales y las personas de confianza". Israel y Rounds (1987) entienden por red social a "aquel conjunto de contactos personales a través de los cuales el individuo mantiene su identidad social y recibe apoyo emocional, ayuda material, servicios, información, etc." Vaux A (1988) lo conceptualiza 
como un metaconstructo en el que se incluyen elementos como los recursos de la red de apoyo, las conductas de apoyo y las evaluaciones del mismo. Hobfoll y Stoke (1988) mencionan que las redes de apoyo social son las "interacciones o relaciones sociales que ofrecen a los individuos, asistencia real o un sentimiento de conexión a una persona o grupo que se percibe como querido o amado". Un año mas tarde Jylhä y Aro (1989) y Hanson, Isacsson, Janzon y Lindell (1989) consideran que el apoyo social puede ser definido como "el apoyo recibo o la interacción entre las personas, sean estos familiares, amigos, vecinos y miembros de organizaciones sociales, ya sea de tipo espiritual, emocional, instrumental e informativo. Bravo M. (1989) opina que el apoyo social es una interacción que se da entre los humanos y en las que se intercambian recursos tanto sociales, como emocionales, instrumentales y recreativos. Además Lin y Ensel (1989) lo refiere como el conjunto de provisiones expresivas o instrumentales (percibidas o recibidas) proporcionadas por comunidades, redes sociales y personas de confianza, sin soslayar que estas provisiones se pueden producir en situaciones cotidianas o de crisis. Similares a los conceptos anteriores, Frey M. (1989) define al apoyo social como las "transacciones interpersonales que incluyen la expresión de afecto positivo de una persona hacia la otra, la afirmación o respaldo de los comportamientos de otra persona, percepciones o puntos de vista expresados y la entrega de ayuda material o simbólica a otra”.

\section{Década de los noventa}

En esta década nace un nuevo concepto sobre las redes de apoyo social con Lemos y Fernández (1990). Ellos definen a la red como "una serie de círculos internos y externos, concéntricos en donde la familia nuclear constituye el círculo más pequeño, los familiares y los amigos dos más, y, tal vez los compañeros de trabajo y otras personas el círculo exterior". En ese mismo año Vaux A. (1990) considera que el apoyo social es un proceso complejo y dinámico, que parte de las transacciones que se dan entre los individuos de la red como respuesta a las demandas de ese contexto y Castañeda (1990) refiere que la red social es un conjunto de contactos que dan identidad social a los individuos, apoyo de diferentes tipos e influye en la búsqueda de ayuda que hacen las personas. Sin embargo, Sánchez A. (1991), refiere el apoyo social es algo personal y subjetivo, mientras que la de redes de apoyo social analizan la estructura social que la componen, siendo esta más objetiva. Bowling y Browne también en el año de 1991, lo conceptualizan como "el conjunto de transacciones emocionales y materiales no regladas entre los individuos que componen la red social. Según menciona Lozares C (1993) "el apoyo social se relaciona a la red social, como un conjunto bien definido de actores, individuos, grupos, organizaciones, comunidades, sociedades globales, entre otros, que están vinculados unos a otros a través de relaciones sociales" y Dabas (1993) como "una fuente de apoyo social que permite el afrontamiento de las necesidades humanas". Un año mas tarde Cornes JM (1994) lo define como "el acceso directo o indirecto a la utilización formal o informal de personas, grupos y organizaciones para reducir lo máximo posible el sentimiento de 
aislamiento, soledad e indefensión de los individuos". En ese mimo año Buendía J. (1994), sugiere que la red social es "el entramado de relaciones sociales en que las personas están inmersas”, mientras que Saidón O. (1995), describe que el término de red, en ocasiones, actúa de manera organizacional y en otras como una función o movimiento social. Rodríguez J. (1995) lo conceptualiza como el conjunto de personas y/o grupos que interaccionan entre sí, así como la estructura de tales interrelaciones.

Partiendo de la segunda mitad de esta década, Barrón A (1996) define al apoyo social como las transacciones que se realizan entre los individuos que componen la red social de una persona, mientras que Sluzki (1996) refería por red social a "toda persona del entorno personal de la mujer con quienes mantiene alguna relación y que ofrecen apoyo, sea éste positivo o negativo". Bronfembrenner (1996) define a la red social como un "sistema de interacción secuencial formada por personas que pueden apoyar, aún sin que la persona en desarrollo esté presente". Abello, Madariaga y Hoyos (1997) mencionan que la red social "es el campo relacional total de una persona y que tiene por común una representación espacio-temporal". Castro, Campero y Hernández (1997) mencionan que el apoyo social se define como la "totalidad de recursos provistos por otras personas". En el mismo año Hupcey y Morse, Campero y Hernández (1997), refieren al apoyo social como un concepto multidimensional asociado a las relaciones sociales y a su influencia ante el estrés y a la salud en general.

Posterior a ello, Sánchez E. (1998) define al apoyo social como "la cantidad y calidad de las relaciones sociales o como recursos emocionales y físicos que nos dan los demás para afrontar situaciones". Por último, Pérez A.M. (1999) sugiere que las redes de apoyo social son "los lazos directos e indirectos que unen a un grupo de individuos según criterios definidos, como la relación de parentesco, de amistad o de conocimiento".

\section{Primera década del dos mil}

Son también diversos los estudios publicados en esta década, sin embargo se hace necesario recalcar que algunos de los autores emplean los conceptos que sobre apoyo social y las redes de apoyo han sido estructurados por otros autores, como es el caso de los estudios de Aranda, Pando, Flores y García (2001) en el que coinciden en que el apoyo social es una variable imprescindible en el análisis del proceso apoyo social/redes de apoyo y salud-enfermedad, retomando el concepto de Cassel J. (1976) como el proceso en que se dan las transacciones entre las personas, agregando lo que por su parte Cobb S. (1976) define por red social, como la instancia mediadora en la que se brinda apoyo social de tipo emocional e informacional, para explicar tal relación. También Barrón y Sánchez (2001) retoman el concepto de Lin, Den y Ensel (1986) "provisiones instrumentales y/ expresivas, reales y percibidas, aportadas por la comunidad, redes sociales y amigos íntimos".

En el 2002 Andrade y Vaitsman refieren que el apoyo social consiste "en las relaciones de intercambio, que implican obligaciones mutuas y los lazos de de- 
pendencia mutua que puede ayudar a crear el sentido de la coherencia y el control de la vida, que benefician a la salud de las personas".

Huenchuan y Sosa (2002) mencionan que Guzmán J. (2002) define al apoyo social como "una practica simbólica-cultural que incluye el conjunto de relaciones interpersonales que integran a una persona con su entorno social y le permite mantener o mejorar su bienestar material, físico y emocional”. Guzmán y Huenchuan (2003), retoman el concepto de Kahn y Antonucci (1980); Bernal, Maldonado y Scharrón (2003) emplean el concepto propuesto por Thoits PA (1982).

Por su parte, Sánchez E. (2004) lo define como "producto de las relaciones sociales, de intercambios individuales regidos por principios psicológicos o como una propiedad emergente de las relaciones sociales" y, Ander E (2004), como "toda acción, conducta o comunicación que tiene el propósito de proteger, auxiliar o ayudar -a otro u otros- a afrontar situaciones problemáticas, de tipo individual, grupal y/o social". El apoyo social, para Ander "es humano, existencial y personal".

Calvo y Díaz (2004) citan a Lin, Dean y Ensel (1986) y a Gottlieb BH (1983). Además Barrón A (2004) y Arias y Barrón (2008), retoman el concepto de la década de los setentas sobre apoyo social. Dabas en el 2006, retoma su concepto sobre las redes de apoyo social propuesto en 1993.

Yanguas y Leturia (2006) consideran que el apoyo social es el conjunto de relaciones sociales, entre las que destacan las familiares, que proveen al sujeto de afecto, ayuda, imagen positiva, información, etc., y que es, al mismo tiempo, un mediador importante en el proceso de afrontamiento de los acontecimientos estresantes como las pérdidas, las enfermedades, los problemas económicos, etc.

Tsibidaki y Tsamparli (2007) citan a McCubbin y Patterson (1981) los cuales definen al apoyo social como "una estrategia externa importante para salir adelante". Topa y Morales (2007) retoman el concepto propuesto por Cohen y Wills en 1985 b, en donde se demuestra que el apoyo social es "una variable que reduce los efectos negativos del estrés en las personas". Pedro, Rocha y Nascimento (2008) mencionan que la red social puede ser entendida como una "red de relaciones en el que se permite que los recursos fluyan a través del apoyo de los bonos". Años más tarde Ponce y cols. (2009) mencionan que el apoyo social "es un proceso interactivo en donde se obtiene ayuda emocional, instrumental y afectiva de la red social que nos rodea, teniendo un efecto protector sobre la salud y amortiguador del estrés que supone una enfermedad". Perilla y Zapata (2009) describen que el apoyo social se utiliza para designar "una característica abstracta de las personas, conductas, relaciones o sistemas sociales, por lo que se le considera más como un metaconcepto que un concepto susceptible de definición y medición".

Fernández y Manrique (2010) hacen mención de estudios de esta época los cuales describen ambas variables de estudio (Mella y cols., 2004; Finfgeld-Connett, 2005) en el que el apoyo social es el "proceso interpersonal de abogacía que está centrado en el intercambio recíproco de información en un contexto específico, el de negociación entre los proveedores y los receptores, a través de la seguridad, 
refuerzo, afirmación, validación y estímulo, bajo una atmósfera de respeto positivo incondicional y cuidado, el cual incluye expresiones de comprensión, respeto, empatía, compasión y amor. En este mismo año vemos publicados una variedad de estudios que enfatizan la importancia de ambos variables, retomando conceptos ya estructurados, entre ellos están los de Rodríguez y cols. (2010) quienes retoman los conceptos de Lin, Simeone y Enzel (1979) y de Bowling (1991). Aranda y cols. (2010) retoman el concepto de Cassel, Caplan y Cobb (1976); Rodríguez (1995), Barrón A (1996), Oliveira y cols. (2010) retoman el concepto de Bronfenbrenner (1996), Fernández y Manrique (2010) mencionan una diversidad de autores como Kahn (1979), Frey (1989), Lozares (1993), Mella y cols. (2004), Finfgeld-Connett (2005), etc. Por su parte Ávila (2010) retoma los conceptos de Dabas (1993, 2006) sobre las redes de apoyo. Ramos y Salinas (2010) retoman el concepto de Yanguas y Leturia (2006). Navarro (2010) retoma el concepto de Castañeda (1990) y Fernández, Díez de la Cortina, Malpica y Hamido (2010) retoman el concepto de Lin y Ensel (1989).

También en este año se hace mención de que el apoyo social es "la ayuda que recibe el individuo de otros en situaciones adversas" (González y Restrepo, 2010) y que las redes sociales de tipo informal "son aquellas que impulsan y propician los contactos personales a través de los cuales el individuo mantiene su identidad social y recibe apoyo emocional, espiritual ayuda material y servicios de información (Hernández, Carrasco y Rosell, 2010).

Un nuevo concepto aparece también en esta época en cuanto a apoyo social y redes de apoyo social, se trata de aquellas relaciones "online" que como dicen los autores "se mantienen con una base relativa de sinceridad y confianza". Se trata de una red que "mantiene y potencializa las relaciones sociales de toda la vida". Las redes sociales "online" pueden "significar un acercamiento renovado al mundo social” (Fuente, Herrero y García, 2010).

\section{CONCLUSIONES}

Como se puede observar tras este recorrido, han sido numerosos los conceptos y abordajes sobre el tema, en los que existe aceptación en el papel protector de las mismas y en las funciones y actividades correspondientes tanto del apoyo social como de las redes de apoyo social, sin embargo, aún se observa que faltan explicaciones sobre su proceso. Sobre toda esta multidimensionalidad sobre los conceptos se manifiesta que el apoyo social incluye una serie de aspectos estructurales y funcionales derivados del mantenimiento de las relaciones sociales.

Además, la proliferación de reportes que tratan de evaluar la relación entre salud y el apoyo social incluidas en estos las redes de apoyo social, tratan de explicar por un lado su efecto amortiguador, y por otro, los efectos directos que el apoyo social gestiona al reducir las consecuencias que tiene para la salud un acontecimiento estresante, ello con la finalidad de asegurar se propicien condiciones adecuadas hacia una mejora en la calidad de vida del individuo. 
Por otro lado, no hay que olvidar que una parte esencial de las redes son los intercambios entre las personas, sean de tipo material, instrumental, emocionales, etc., todos ellos son intercambios que influirán en el grado de satisfacción de las necesidades de las personas. Aunado a lo anterior, se alcanzan a distinguir dos tipos de redes: la informales (en donde las interacciones existentes, se dan principalmente por la familia, cónyuge, hijos, hermanos, familiares y amistades), y las formales (en el que las interacciones que se brindan se dan principalmente por grupos, organizaciones, centros sociales, centros de salud, etc), y en el que, todas ellas, en menor o mayor grado, son importantes y necesarias ante cualquier situación, sea esta crítica o no.

En fin, una de las ventajas de este trabajo es que, de la gran variedad de conceptos plasmados más de alguno puede ser utilizado en estudios futuros, claro está que ello dependerá de los objetivos y finalidades de la investigación. Para el presente trabajo se entenderá por apoyo social a las interrelaciones que se dan entre las personas, con conductas que también se relacionan entre sí como el demostrar cariño e interés, escuchar, prestar objetos materiales, ayudar económicamente, cuidar de alguien, dar afecto, sentirse amado, dar consejo, aceptarse, satisfacerse, informarse, entre algunas, y por redes de apoyo social sean estas formales e informales a las instancias que moderan esas conductas de apoyo, además de concluir que, con la falta de apoyo o con su deficiencia, la mala salud está presente, o al menos es más probable, y que las respuestas negativas generadas, provocarán en el individuo consecuencias tanto en su ámbito familiar, social y laboral.

\section{REFERENCIAS BIBLIOGRÁFICAS}

Abello, R., Madariaga, C., Hoyos, O. L. (1997). Redes sociales como mecanismo de supervivencia: un estudio de casos en sectores de extrema pobreza. Revista Latinoamericana de Psicología, 29: 115-137.

Andrade, G. R. B., Vaitsman, J. (2002). Apoio social e redes: conectando solidariedade e saúde. Ciênc Saúde Coletiva, 7(4): 925-34.

Ander, E. (2004). Los grupos de autoayuda y el apoyo social. Ergon, Madrid.

Aranda, C., Pando, M., Flores, M. E., García, T. (2001). Depresión y redes sociales de apoyo en el adulto mayor institucionalizado de la zona metropolitana de Guadalajara, Jalisco. Rev Psiquiatría, 28: 69-74.

Aranda, B. C., Pando, M. M. (2010). Edad, síndrome de agotamiento profesional (Burnout), apoyo social y autoestima en agentes de tránsito, México. Rev Colomb Psiquiat, 39(3): 510-522.

Arias, A., Barrón, A. (2008). El apoyo social en la predicción a corto y medio plazo de la permanencia del voluntariado socioasistencial. Psicothema, 20: 97-103.

Ávila, T. J. H., Madariaga, O. C. (2010). Redes personales de apoyo en individuos desmovilizados del conflicto armado. Psicología desde el Caribe, 25:179-201. 
Barnes, J. A. (1954). Class and Committees in a Norwegian Island Parish. Human Relations, 7: 39-58.

Barrón, A. (1996). Apoyo social. Aspectos teóricos y aplicaciones. Madrid, España: Siglo XXI de España editores.

Barrón, A. (2004). Estrés, apoyo social y trastornos cardiovasculares. Jano, 68: 93-94.

Barrón, A., Sánchez, E. (2001). Estructura social, apoyo social y salud mental. Psicothema, 13: 17-23.

Bell, R. A., Leroy, J. B., Vitalo, R. (1982). Evaluating the mediating effects of social support upon life events and depressive symptoms. Journal of Community Psychology, 10: 325-340.

Berkman, L. F. (1984). Assesing the physical health effects of social networks and social support. Ann Rev Public Health, 34: 1-9.

Bernal, G., Maldonado, M. M., Scharrón, M. R. (2003). Desarrollo de una Breve Escala de Apoyo Social: Fiabilidad y validez en Puerto Rico. Revista Internacional de Psicología Clínica y de la Salud, 3: 251-264.

Bott, E. (1995). "Urban Families: Conjugal Roles and Social Networks". Human Relations, 8: 345-384.

Bowling, A., Browne, P. D. (1991). Social networks, health, and emotional well-being among the oldest old in London. J Gerontol Soc Sci, 1: 520-532.

Bowly, J. (1969). Attachment and Loss. Attachment, Londres, Hogarth Press.

Bravo, M. (1989). Las redes de apoyo social y las situaciones de desastre: un estudio de la población adulta en Puerto Rico. Unpublished Dissertation, University of Puerto Rico, Río Piedras.

Bronfenbrenner, U. (1996). A ecologia do desenvolvimento: experimentos naturais e planejados. Porto Alegre (RS): Artes Médicas.

Buendía, J. (1994). Envejecimiento y psicología de la salud. España: Siglo Veintiuno de España S.A.

Calvo, F., Díaz, M. D. (2004). Apoyo social percibido: características psicométricas del Cuestionario Caspe en una población urbana geriátrica. Psicothema, 16: 570-575.

Caplan G. (1974). Support systems and community mental health: lectures on concept development. New York: Behavioral Publications.

Caplan, G., Killilea, M. (1976). Support systems and mutual help: Multidisciplinary explorations. Grune and Stratton: New York.

Caplan, R., Robinson, E., French, J., Caldwell, J., Shinn, M. (1976). Adhering to medical regimens: Pilot experiments in patient education and social support. Ann Arbor: Institute for Social Research, The University of Michigan.

Cassel, J. (1974). Psychosocial processes and «stress»: Theoretical formulation. International Journal of Health Services, 4: 471-482. 
CONCEPTUALIZACIÓN DEL APOYO SOCIAL Y LAS REDES DE APOYO SOCIAL

Cassell, J., Caplan J., Cobb, S. (1976). The contribution of the social environment to host resistance. American Journal of Epidemiology, 104: 107-123.

Castañeda, C. I. (1990). Utilización de servicios de atención prenatal: influencia de la morbilidad percibida y de las redes sociales de ayuda. Salud Pública Mex, 32:419-429.

Castro, R., Campero, L., Hernández, B. (1997). La investigación sobre apoyo social en salud: situación actual y nuevos desafíos. Revista de Saude Pública, 31: 425-435.

Cobb, S. (1976). Social support as a moderator of life stress. Psychosomatic Medicine, 38: $300-314$.

Cohen, S., Syme, L. (1985a). Issues in the study and application of social support. In: Cohen, S., Syme, S. L.(ed.), Social support and health. New York: Academic Press.

Cohen, S., Wills, T. (1985b). Stress, social support and the buffering hypothesis. Psychological Bulletin, 98, 310-357.

Cornes, J. M. (1994). El apoyo social; su relevancia en la práctica psiquiátrica. Revista Psiquiátrica. Facultad Medicina Barna, 21: 147-152.

Dabas, E. (1993). Red de redes. Las prácticas de la intervención en redes sociales. Buenos Aires: Paidós.

Dabas, E. (2006). Viviendo redes. En Dabas, E. (comp). Viviendo redes. Experiencias y estrategias para fortalecer la trama social. Argentina: Ediciones Ciccus.

Da Silva, P. I. C., Melo, R. S. M., Castanheira, N. L. (2008). Apoio e rede social em enfermagem familiar revendo conceitos. Rev Latinoam Enfermagem, 16(2): 324-27.

Finfgeld, C. D. (2005). Clarification of social support. Journal of Nursing Scholarship, 37(1):4-9.

Fernández, A. R., Manrique, A. F. G. (2010). Rol de la enfermería en el apoyo social del adulto mayor. Enfermería Global, 19:1-9.

Fernández, M. J. M., Díez de la Cortina, D., Malpica, B. M. J., Hamido, M. A. (2010). Relación entre el apoyo social, la satisfacción vital y las expectativas de futuro en menores acogidos es centros de protección. Electronic Journal of Research in Educational Psychology, 8(2):643-654.

Fuente, A., Herrero, J., Gracia, E. (2010). Internet y apoyo social: Sociabilidad online y ajuste psicosocial en la sociedad de la información. Acción Psicológica, 7(1): 9-15.

Frey, M. (1989). Social support and health: A theoretical formulation derived from King's Conceptual Framework. Nursing Science Quarterly, 2(3):138-148.

González, N. (2001). Acercamiento a la literatura sobre redes sociales y apoyo social. Revista Cubana de Psicología, 18: 134-141.

González, Q. J. C., Restrepo, C. G. (2010). Prevalencia de felicidad en ciclos vitales y relación con redes de apoyo en población colombiana. Rev Salud Pública, 12(2): 228-238.

Gottlieb, B. H. (1983). Social support strategies: Guidelines for mental health practice. Beverly Hills, Sage Publications. 
Guzmán, J. M. (2002). Redes de apoyo social a personas mayores: Marco conceptual. Documento presentado en la Reunión de Expertos en Redes de Apoyo Social a Personas Mayores, CEPAL, Santiago de Chile, del 9 al 12 de diciembre.

Guzmán, J. M., Huenchuan, S. (2003). Redes de Apoyo Social de las personas mayores: Evidencias Para América Latina. Ponencia presentada en el III Congreso Panamericano de Gerontología "El Desafío en Envejecer en las Américas", Mar del Plata - Argentina, 30 de abril al 3 de mayo.

Hanson, B. S., Isacsson, S. O., Janzon, L., Lindell, S. E. (1989). Social network and social support influence mortality in elderly men. The prospective population study of «Men born in 1914» Malmö, Sweden. Am J Epidemiol, 130:100-11.

Hernández, M. G., Carrasco, G. M. R., Rosell, C. F. (2010). Titulo: Evaluación de las principales redes de apoyo informal en adultos mayores del Municipio Cerro. Publicación Periódica de Gerontología y Geriatría, 5(1): 1-11.

Hobfoll, S. E., Stokes, J. P. (1988). The process and mechanics of social support. In S. Duck (Ed.), Handbook of personal relationships (pp. 497-517). New York: John Wiley \& Sons, Ltd.

Huenchuan, S., Sosa, Z. (2002). Redes de apoyo y calidad de vida de personas mayores en Chile. Ponencia presentada en la Reunión de Expertos en Redes de Apoyo Social, el rol del Estado, la Familia y la Comunidad, organizada por CELADE-División de Población de la CEPAL. Santiago de Chile, 9 al 12 de diciembre.

Hupcey, J., Morse, J. (1997). Can a professional relationship be considered social support? Nursing Outlook, 45(6):270-276.

Israel, B. A. (1982). Social networks and health status: linking theory, research, and practice. Patient counseling and health education, 4: 65-79.

Israel, B. A., K. Rounds. (1987). Social Networks and Social Support: A Synthesis for Health Educators. Advances in Health Education and Promotion, 2: 311-351.

Johnson, J. I H., Sarason, I. G. (1979b). Recent Developments in Research on Life Stress. En V. Hamilton y D. M. Warburton (Eds), Human Stress and Cognition: An information processing approach, London: Wiley.

Johnson, J. I. H., Sarason, I. G. (1979a). Moderator variabels in life stress research. En I.G. Sarason y C.D. Spielberger (Eds.), Stress and anxiety (Vol. 6). Washington, D.C: Hernisphere.

Jylhä, M., Aro, S. (1989). Social ties and survival among the elderly in Tampere, Finland. Int J Epidemiol, 18: 158-164.

Kahn, R. L. (1979). Aging and social support. Riley M, Ed. Aging from birth to death. Boulder: Westview.

Kahn, R. L., Antonucci, T. C. (1980). Convoys over the life course: Attachment, roles and social support. In: Baltes, P., Brim, O. (Eds.), Life Span Development and Behavior. 3: 253-286. San Diego, CA: Academic Press. 
Kahn, R. L., Antonucci, T. C. (1981). Convoys of social support: A life-course approach. En J.G. March., S.B. Kiesler, J.N., Morgan, V. K. Oppenheimer (eds.), Aginf: Social Change. Londres: Academic Press.

Kaplan, B. H., Cassel, J. C., Gore, S. (1977). Social support and Health. Med Care, 15: 47-58.

Lin, N., Ensel, W. (1989). Life stress and health: stressors and resources. Am Sociol Rev, 54:382-399.

Lin, N., Dean, A., Ensel, W. M. (1986). Social support, life events and depressions, Nueva York, Academic Press. En Barrón, A. 1996. Apoyo social. Aspectos teóricos y aplicaciones. Siglo Veintiuno de España Editores, Madrid.

Lin, N., Simeone, S. R., Ensel, W. M., Kuo, W. (1979). Social ad empirical test. Journal of Health and Social Behavior, 20: 108-119.

Maguire, L. (1980). "The interface of social workers with personal networks", Social Work with Groups, Vol. 3, Nueva York, The Haworth Press.

Mella, R., González, L., D’appolonio, J., Maldonado, I., Fuenzalida, A., Díaz, A. (2004). Factores Asociados al Bienestar Subjetivo en el Adulto Mayor. Psykhe, 13(1):79-89.

Navarro, G. A. M., Rodríguez, G. R. (2010). Redes de apoyo en el anciano por medio del genograma y el ecomapa. Aten Fam, 17(1):19-21.

Oliveira Di Primio, A., Schwartz, E., Machado, B. V. L., Burille, A., Vestena, Z. J. G., Machado, F. A. (2010). Rede social e vínculos apoiadores das famílias de criancas com Cáncer. Contexto Enferm, 19(2): 334-42.

Pearlin, L. I., Lieberman, M. A., Menaghan, E. G., Mullan, J. T. (1981). The Stress Process. Journal of Health and Social Behavior, 22: 337-356.

Perilla, L. L., Zapata, B. C. (2009). Redes sociales, participación e interacción social. Trabajo Social, 11: 147-158.

Pérez, A. M. (1999). Personalidad, afrontamiento y apoyo social. Madrid, UNED-FUE.

Ponce, G. J. M., Velásquez, S. A., Márquez, C.E., López, R. L., Bellido, M. M. L. (2009). Influencia del apoyo social en el control de las personas con diabetes. Index de enfermería: información, 18(4): 224-228.

Ramos, E. J., Salinas, G. R. J. (2010). Vejez y apoyo social. Revista de Educación y Desarrollo, 69-76.

Rodríguez, J. (1995). Psicología Social de la Salud. Editorial Síntesis, Madrid.

Rodríguez, M. A., Ruiz, R. F., Antón, B. J. J., Herrera, J. J., Máiquez, P. A. (2010). A Ottaviano Castillo. ¿Tienen apoyo social los reclusos drogodependientes? Estudio en una prisión andaluza. Rev Esp Sanid Penit, 12: 13-20 13.

Saidón, O. (1995). Las redes: pensar de otro modo. En: Elina Dabas y Dense Najmanovich, comp.: Redes. El lenguaje de los vínculos hacia la reconstrucción y el fortalecimiento de la sociedad civil. Argentina: Ed. Paidós. 
Sánchez, A. (1991). Psicología comunitaria. Bases conceptuales y operativas. Métodos de intervención. Barcelona: Ed. PPU, Colección LCT-1

Sánchez, E. (1998). Apoyo social, integración social y salud mental. Revista de Psicología Social, 13: 537-544.

Sánchez, E. (2004). Collectivize Social Support? Elements for Reconsidering the Social Dimension in the Study of Social Support. The Spanish Journal of Psychology, 7: 124-134.

Sarason, G., Levine, M., Basham, B., Sarason, R. (1983). Assesing social support: The social support questionnaire. Journal of Personality and Social Psychology, 44: 127-139.

Sarason, B.R., Sarason, I.G., Hacker, T.A., Basham, R.B. (1985). Concomitants of Social Support: Social Skills, Physical Attractiveness, and Gender. Journal of Personality and Social Psychology, 49:469-480.

Shumaker, S.A., Brownell, A. (1984). Toward a theory of social support: Closing conceptual gaps. Journal of Social Issues, 40: 11-36.

Shumaker, S. A., Brownell, A. (1985). Toward a theory of social support: Closing conceptual graps. Journal of Social Issues, 40: 11-36.

Sluzki, C. E. (1996). La red social: frontera de la práctica sistémica. Barcelona, Gedisa.

Thoits, P. A. (1982). Conceptual, methodological and theoretical problems in studying social supports as a buffer against life stress. Journal of Health and Social Behavior, 23: $145-159$.

Topa, G., Morales, J. F. (2007). Burnout e identificación con el grupo: el papel del apoyo social en un modelo de ecuaciones estructurales. International Journal of Clinical and Health Psychology, 7: 337-348.

Tsibidaki, A., Tsamparli, A. (2007). Redes de apoyo a las familias griegas con hijos discapacitados en edad escolar. Revista Electrónica de Investigación Psicoeducativa, 12, 52: 283-306.

Vaux, A. (1988). Social and emotional loneliness: The role of social and personal characteristics. Personality and Social Psychology Bulletin, 14: 722-734.

Vaux, A. (1990). An ecological approach to understanding and facilitating social support. Journal of Social and Personal Relationships, 7: 507-518.

Wellman, B. (1981). Applying analysis to the study of support. En B. H. Gottlieb (Ed.). Social Networks and Social Support. London: Sage.

Yanguas J., Leturia FJ. (2006). "Intervención psicosocial en personas mayores". En: Triadó C., Villar F. Psicología de la vejez. Madrid: Alianza Editorial. 